\title{
ANALISE DA MORFOLOGIA DE CARBETOS (NI,TI)C DA SUPERLIGAS DE NIQUEL 718 APÓS PROCESSO TERMOMECÂNICO*
}

Alessandra Vieira Guimarães ${ }^{1}$ Leonardo Sales Araujo ${ }^{2}$

\section{Resumo}

A superliga de níquel 718 é uma superliga endurecida por precipitação, com alta resistência mecânica sob condições extremas e ambientes agressivos. Encontra aplicações como componentes críticos para indústrias aeroespacial, óleo e gas e nuclear. Seu alto teor de elementos de liga promove a ocorrência de diversas fases com consequente impacto nas propriedades. O presente trabalho foca na comparação por análise de imagens na morfologia, fração volumétrica, tamanho médio e distribuição de partículas de carbonetos ( $\mathrm{Nb}, \mathrm{Ti}) \mathrm{C}$ após duas rotas de processamento termomecânico. Sua quantidade, distribuição e morfologia podem ter um papel em processos de degradação como corrosão e fragilização ao hidrogênio. Portanto, é importante saber como se desenvolve sob diferentes rotas de processamento.

Palavras-chave: Superliga 718; Inconel 718; Carboneto; Processamento termomecânico.

\section{ANALYSIS OF (NB,TI)C CARBIDE MORPHOLOGY IN NICKEL-BASE SUPERALLOY 718 AFTER THERMOMECHANICAL PROCESSING}

\section{Abstract}

Nickel base superalloy 718 is a precipitation hardened Ni-base superalloy, with high strength under extreme temperatures and harsh environments. It finds applications as critical components for airspace, oil and gas and nuclear industries. Its high content of allying elements promotes several phases with impact on properties. The present work aims to compare by image analysis the morphology, volume fraction, average size and distribution of $(\mathrm{Nb}, \mathrm{Ti}) \mathrm{C}$ particles after two different routes of thermomechanical processing. Its amount, distribution and morphology can play a role on degradation processes such as corrosion and hydrogen embrittlement. It is, then, important to know how it develops under different processing routes.

Keywords: Superalloy 718; Inconel 718; Carbide; Thermomechanical processing.

1 Estudante de Engenharia de Materiais, Universidade Federal Rural do Rio de Janeiro, Rio de Janeiro, RJ, Brasil.

2 Engenheiro Metalúrgico, DSc, Professor, Programa de Engenharia Metalúrgica e de Materiais, COPPE/UFRJ, Rio de Janeiro, RJ, Brasil. 


\section{INTRODUÇÃO}

O principal estímulo para o desenvolvimento das superligas surgiu da necessidade de se obter materiais de alta resistência, com a capacidade de sustentar as suas propriedades mecânicas sob altas temperaturas e em ambientes severos, como turbinas a gás e motores de turbinas de aeronaves[1-4].

As superligas podem ser agrupadas por seus elementos de transição preponderantes - Ni, Fe ou Co - que são responsáveis por formar uma matriz cúbica de face centrada (Fe e Co são objetos de transformações alotrópicas na estrutura cfc em alta temperatura). $\mathrm{Cr}$ é adicionado principalmente para dar resistência à oxidação, e outros elementos de liga como $\mathrm{Al}, \mathrm{Ti}, \mathrm{Nb}, \mathrm{W}, \mathrm{Mo}, \mathrm{Ta}$ dão oendurecimento por solução sólida e / ou de endurecimento por precipitação. Como resultado, aumento da força e estabilidade química são mantidas em temperaturas acima de $540^{\circ} \mathrm{C}[1,2,5]$.

Há um vasto campo de aplicações para superligas, especialmente como componentes críticos para as indústrias do espaço aéreo, petroquímicas e de geração de energia, como Estudantelâminas de turbina e discos, válvulas, molas ou parafusos para reatores nucleares ou vasos de tubulação e de reação para plantas petroquímicas [2].Em particular, a superliga de $\mathrm{Ni} 718$ apresenta um excelente conjunto de propriedades, como alta resistência e tenacidade em temperaturas criogênicas até $650^{\circ} \mathrm{C}$, e resistência a ambientes agressivos como água marinha, motor de ônibus espacial ou o núcleo de reatores nucleares de PWR [6-8] , além de poder ser fabricado por diferentes vias como fundição, forjamento, metalurgia do pó ou manufatura aditiva [2,9].Suas excelentes propriedades advém do controle de sua microestrutura. A alta resistência mecânica da liga é induzida primordialmente pela precipitação das fases endurecedoras e metaestáveis $\gamma^{\prime}\left(\mathrm{Ni}_{3}(\mathrm{Al}, \mathrm{Ti}), \mathrm{L}_{2}\right)$ e $\gamma^{\prime \prime}$ (Ni3Nb, $\mathrm{D0}_{22}$ ), sendo a última mais efetiva. Além destas, as principais fases usualmente observadas são a $\delta\left(\mathrm{Ni} 3 \mathrm{Nb}, \mathrm{DO}_{\mathrm{a}}\right)$ e carbonetos $(\mathrm{Nb}, \mathrm{Ti}) \mathrm{C}[2,10]$.

Especificamente, com relação aos carbonetos (Nb,Ti)C, esses estão sempre presentes na liga e normalmente se apresentam como agrupamentos de partículas alinhados a direção principal de deformação da liga. Apesar dessa fase não contribuir de forma significativa para o endurecimento da liga podem atuar de forma significativa em fenômenos como corrosão ou como fragilização ao hidrogênio $[9,11]$.

Com isso, o objetivo do presente trabalho é determinar a fração volumétrica de ( $\mathrm{Nb}, \mathrm{Ti}) \mathrm{C}$ na liga, assim como o tamanho médio de partículas e sua morfologia após diferentes rotas de processamento termomecânico.

\section{MATERIAIS E MÉTODOS}

Amostras de chapas de superliga 718 foram processadas termomecanicamente utilizando duas rotas. Na primeira rota, a amostra foi submetida a4 etapas de laminação a quente, com $20 \%$ de deformação por passe, a $1050^{\circ} \mathrm{C}$. Entre os passes e após o último, o material permaneceu no forno por $1 \mathrm{~h}$. Tal amostra foi denominada "LQ. Para a segunda rota, realizaram-se 4 etapas de laminação a frio, com $20 \%$ de deformação poretapa, seguido de recozimento a $1050^{\circ} \mathrm{C}$ por 1 hora. Tal amostra foi denominada "ECG".

Para a observação da microestrutura das amostras, as mesmas foram preparadas por métodos convencionais de metalografia, comocorte, embutimento em baquelite, lixamento com lixas de 100, 240, 320, 400, 600, 1200 \#. O polimento foi realizado 
com um pano de $3 \mu$, seguindo o polimento com um pano $1 \mu$ com pasta de diamanteem uma máquina automática com carga baixa para evitar a quebra das partículas e arranhões. As amostras foram submetidas à limpeza em ultra-som, durante 5 minutos.

De forma a verificar a existência de outras fases além da matriz $\gamma$ e o carboneto $(\mathrm{Nb}, \mathrm{Ti}) \mathrm{C}$ análises por difração de raios- $X$ foram realizadas nas amostras LQ e ECG.

As imagens foram obtidas por microscopia eletrônica de varredura, em modo retroespalhado, com aumentos de 100x e 200x, afim de encontrar um balanço entre representatividade da microestrutura e resolução para identificação dos carbonetos. Cabe ressaltar que os campos foram definidos de maneira aleatória, de forma a evitar um viés na análise.A análise das imagens foi baseada em análise do histograma de 8 bits, binarização (separando as partículas de carbonetos da matriz). Para tal, foi utilizado o software Image J. Com isso, as frações de fase e tamanho médio de partículas foram determinadas.Os resultados das análises para cada imagem foram consolidados e realizada uma análise estatística com o software PSPP.

Para averiguar os resultados das análises de imagem, foram realizadas simulações com o software Thermocalc, usando a base de dados TTNI8 [12]. Foi determinada a fração máxima em peso do carboneto, sendo depois convertida para fracão volumétrica.

\section{RESULTADOS E DISCUSSÃO}

A análise de difração de Raio-X (Figura 3.b) confirmou que a temperatura escolhida para o processamento efetivamente solubilizou as fases $\gamma^{\prime}, \gamma^{\prime \prime}$ e $\delta$, deixando apenas a matriz $\gamma$ e o carboneto $(\mathrm{Nb}, \mathrm{Ti}) \mathrm{C}$. A Figura 1 mostra o espectro de raios $X$ da liga nas condições LQ e ECG. No espectro das amostras ECG, não foi possivel discernir o pico.

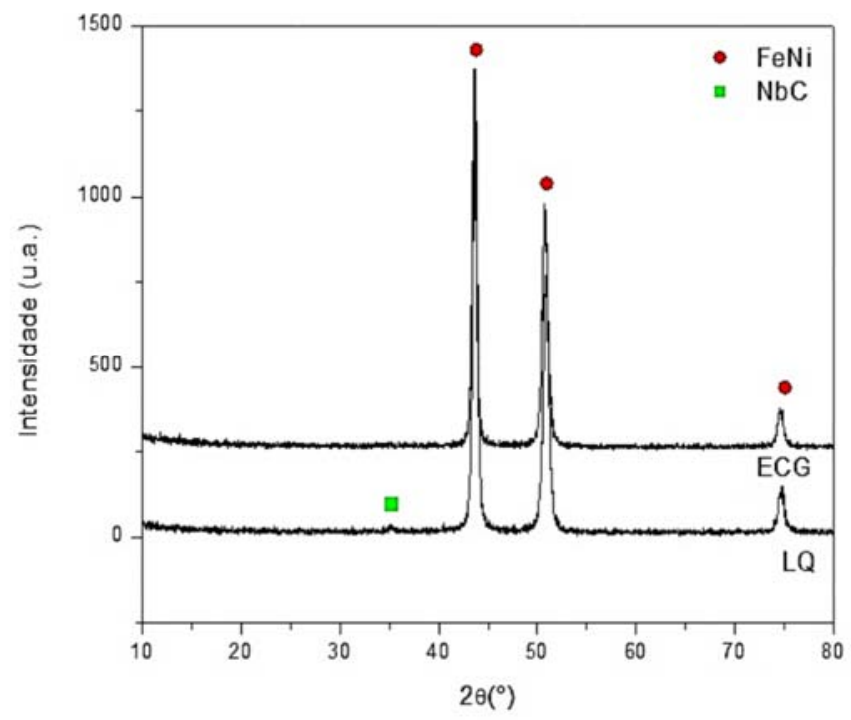

Figura 1. (espectro de raios-X das amostras LQ e ECG. É possivel observar picos apenas para a matriz g e o carboneto $(\mathrm{Nb}, \mathrm{Ti}) \mathrm{C}$

A análise das imagens revelou os carbetos alinhados à direção de laminação, como mostrados na Figura 2. 


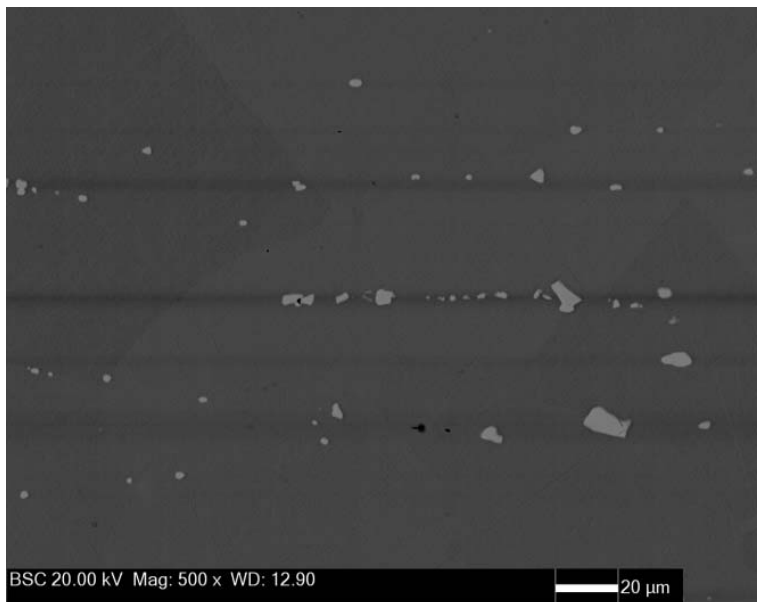

Figura 2. Carboneto (Nb,Ti)C (regiões cinza claro) alinhado a direção de processamento (sentido horizontal).

A Figura 3 mostra duas images, cada uma relativa a uma condição de processamento: LQ e ECG.

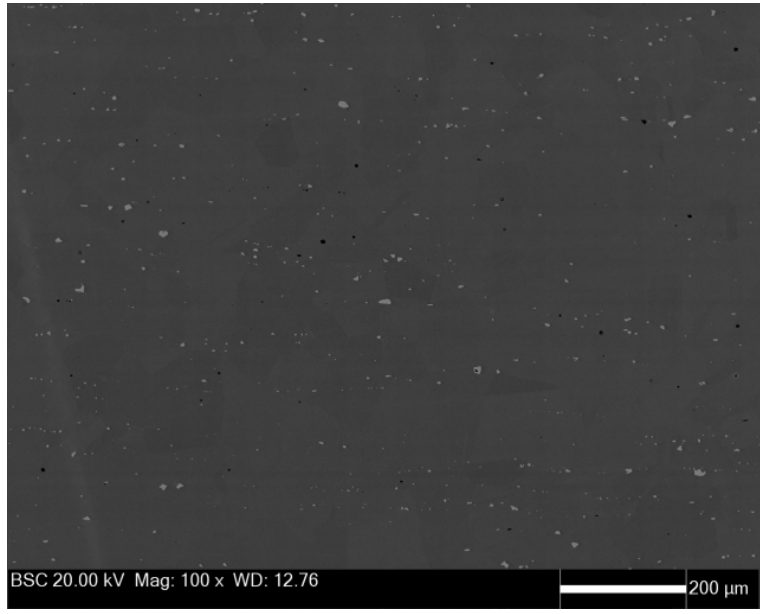

(a)

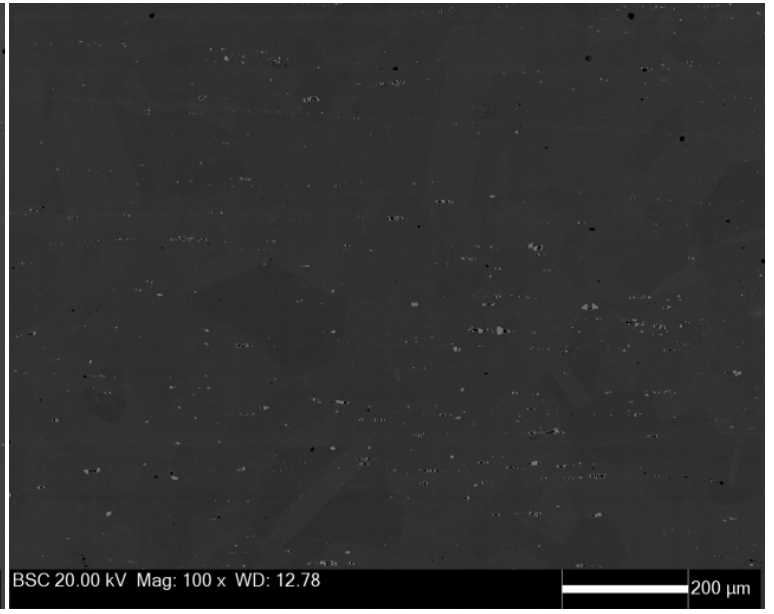

(b)

Figura 3. Imagens no MEV de: (a) Amostra LQ e; (b) Amostra ECG, mostrando os carbetos (Ni,Ti)C enfilheirados (manchas brancas) na direção de laminação.

A análise comparativa da fração de carbonetos em aumentos nominais de 100x e 200x não apresentou diferenças significativas nos valores médios, indicando que uma imagem com aumento nominal de 100x apresentou resolução suficiente para a clara detecção dos mesmos, com a vantagem de ser mais representativa em relação a um maior aumento nominal.

Os resultados relativos as frações calculadas para cada imagem foram reunidos e conduzida uma análise estatística baseada em teste $\mathrm{T}$ para independência das médias. Foi usado um intervalo de confiança de $95 \%$ e analisadas as frações de área ocupada pelos carbonetos e o tamanho médio das partículas. A Tabela 1 apresenta os resultados da análise estatística. Tal análise mostrou que houve diferença nas médias tanto da fração de fase quanto no tamanho médio das partículas entre as diferentes rotas de processamento. As amostras LQ apresentaram mair fração de área e maior tamanho de partículas que as amostras ECG. Ambos os resultados são esperados e podem ser explicados considerando a evolução dos processos. O processamento identificado como ECG baseia-se em 
sucessivas etapas de deformação a frio, o que torna mais provável a que as partículas de carbonetos (as quais são duras) sejam mais fragmentadas durante o processo de deformação a frio. No caso das amostras LQ, estas foram deformadas a quente $\left(1050^{\circ} \mathrm{C}\right)$, reduzindo a fragmentação das mesmas e, consequentemente, resultando em maior tamanho médio de partículas em relação às amostras ECG. Além disso, a própria deformação da matriz permite a maior dispersão dessas partículas na matriz, comportamento também notado nas análises de imagens. A menor fração volumétrica nas amostras ECG pode ser relacionada com o fato de partículas mais fragmentadas terem maior facilidade de arrancamento durante o polimento, o que resulta em uma menor fração identificada das mesmas na análise de imagens.

Tabela 1. valores médios e desvios das análises de fração de área e tamanho de partículas para as amostras LQ e ECG.

\begin{tabular}{ccc}
\hline Amostra & $\begin{array}{c}\text { Fração de área de } \\
(\mathrm{Nb}, \mathrm{Ti}) \mathrm{C}\end{array}$ & $\begin{array}{c}\text { Tamanho médio de } \\
\text { partículas }(\mu \mathrm{m})\end{array}$ \\
\hline LQ (laminada a quente) & $0,56 \pm 0,06$ & $12,88 \pm 0,76$ \\
$\begin{array}{c}\text { ECG (laminada a frio e } \\
\text { tratada termicamente) }\end{array}$ & $0,47 \pm 0,03$ & $9,57 \pm 1,14$ \\
\hline
\end{tabular}

Testes realizados com intervalo de confiança para 95\% de confiabilidade.

A simulação da formação de carboneto com a temperatura mostrou que a fração em massa estimada para essa fase é cerca de $0,38 \%$. A conversão para fração volumétrica foi realizada e encontrado um valor de $0,43 \%$, valor esse próximo àqueles encontrados pela análise de imagem, o que reforça a validade do método de determinação da fração de carbonetos. A Figure 4 mostra o diagrama de resfriamento, mostrando a evolução da fração volumétrica do carboneto com a temperatura

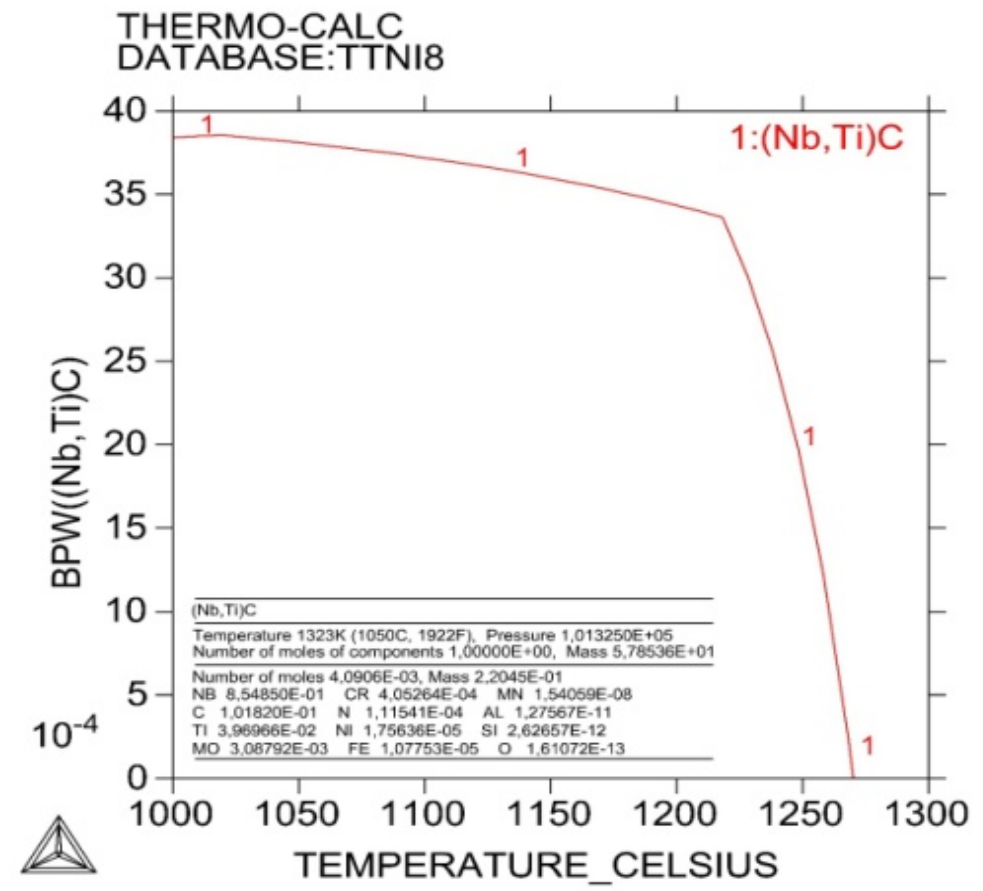

Figura 4. Simulação por Thermocalc da evolução da fração em massa de (Nb,Ti)C com a temperatura. 


\section{CONCLUSÃO}

Baseado nas análises realizadas foi possível concluir que o método de análise de imagens foi eficaz na determinaçao da fração de fase de carbonetos $(\mathrm{Nb}, \mathrm{Ti}) \mathrm{C}$. $\mathrm{O}$ percentual encontrado foi em torno de $0,5 \%$, próximo ao valor determinado por simulação termodinânica $(0,43 \%)$, o que ratifica a metodologia adotada.

Verificou-se com significância estatística ( $95 \%$ de confiabilidade) que tanto a fração de fase quanto o tamanho médio de partícula foi menor para a amostra ECG, a qual foi deformada a frio e várias etapas. Tal fato pode ser relacionado a maior frequência de quebra das partículas duras de carboneto durante o processo de deformaçao a frio, em comparação a deformação a quente das amostras LQ.

Nas amostras LQ os carbonetos ficaram mais dispersamente distribuídas que no caso ECG, possivelmente devido a maior possibilidade de deformação da matriz durante o processo de laminação a quente.

\section{Agradecimentos}

Os autores gostariam de agradecer o apoio financeiro do CNPq e ao Laboratório de Materiais do CEFET/RJ.

\section{REFERÊNCIAS}

$1 \quad$ R. Bowman, "Superalloys: A Primer and History," [Online]. Disponível em: http://www.tms.org/meetings/specialty/superalloys2000/superalloyshistory.html [Acessado emjaneiro de 2015].

2 M. Donachie and S. Donachie, Superalloys: A Technical Guide, Materials Park, $\mathrm{OH}$ : ASM International, 2002.

3 B. Geddes, H. Leon and X. Huang, Superalloys: Alloying and Performance, Materials Park, OH: ASM International, 2010.

4 C. T. Sims, "A history of superalloy metallurgy for superalloys metallurgists," in Fifth International Symposium on Superalloys, 1984.

5 J. R. Davis, Ed., ASM Specialty Handbook: Nickel, Cobalt, and Their Alloys, Materials Park: ASM International, 2000.

6 R. P. Jewet and J. A. Halchak, "The use of alloy 718 in space shuttle main engine," in Superalloys 718, 625 and various derivatives, 1991.

7 A. Rowclife, L. Mansur, D. Hoeler and R. K. Nanstad, "Perspective on radiation effects in nickel-base alloys for applications in advanced reactors," Journal of Nuclear Materials, vol. 392, pp. 341-352, 2009.

8 American Petroleum Institute, API6A718 - Specification of Nickel-base alloy 718 (UNS N07718) for oil and gas drilling and production equipment, American Petroleum Institute, 2004.

9 Q. Jia and G. Dongdong, "Selective laser melting additive manufacturing of Inconel 718 superalloy parts: Densification, microstructure and properties," Journal of Alloys and Compounds, vol. 585, pp. 713-721, 2014.

10 Araujo, L.S., Processamento e caracterização de superliga de níquel 718 para aplicações nucleares, Tese de doutorado, COPPE/UFRJ, 2012;

11 Menezes, L.C. A influência da fase delta no comportamento mecânico e na resistência à corrosão da Superliga Inconel 718, Tese de doutorado, COPPE/UFRJ, 2014;

12 Thermotech Ltd NI-DATA Nickel Superalloys database version 8, (Accessado em14 de março de 2015). 